\title{
Self-Activation or Keying
}

National Cancer Institute

\section{Source}

National Cancer Institute. Self-Activation or Keying. NCI Thesaurus. Code C62844.

Problem associated with the unintended activation of the device, or a device having been unexpectedly turned on during use. 